\title{
Soft X-Ray Spectroscopic Study of a Gas-Puff Z-Pinch Argon Plasma
}

\author{
Seong Ho Kim, Dong-Eon Kim, and Tong Nyong Lee
}

\begin{abstract}
X-ray radiation characteristics of argon plasma produced by a gas-puff $Z$-pinch device were investigated using an X-ray crystal spectrometer, an X-ray diode, and an extreme ultraviolet (XUV) spectrometer. Using a germanium crystal we have observed spectral emission from Ar XVII produced by hot spots at the pinched stage. With the help of a 2-m grazing incidence XUV spectrometer, the spectrum of 30 to $250 \AA$ were obtained. Strong lines from Ar VIII to Ar XIII were observed with a continuum whose peak is around $120 \AA$. The radiation energy in the spectral range is estimated to be about 23 joule which is about $0.6 \%$ of the electrical energy stored in capacitors.
\end{abstract}

Index Terms-Gas-puff Z-pinch, spectroscopy, X-ray.

\section{INTRODUCTION}

$\mathbf{Z}$ -PINCH plasma has been widely studied as an intense pulsed light source in the soft X-ray region for various $\mathrm{X}$-ray applications such as microscopy, lithography [1], and $\mathrm{X}$-ray lasers [2], [3]. Its dynamics have been known to be complicated, and particularly the origin and dynamics of hot spots have attracted many researchers [4]. We have constructed a gas-puff Z-pinch device to study its radiation characteristics. The experiments for the study of hot spots and the estimation of radiation energy in the XUV spectral region were conducted. The $d I / d t$ signal of the discharge was recorded to monitor the reproducibility of pinches. The spectroscopic study of small-scale gas-puff Z-pinch has been done by many researchers [5]-[7], and the energy estimation using vacuum X-ray diode (XRD) arrays was done by Bailey [9], where the spectral profile was deconvoluted from the simultaneous signals of the XRD arrays with different filters.

In this paper, we present the basic diagnostic results obtained using a crystal and an XUV spectrometer, and the estimation of energy radiated in the spectral region of 44 to $110 \AA$ using an XRD where the spectral profile obtained with an XUV spectrometer is used to estimate the energy.

\section{EXPERIMENTAL AND DiAgNOSTIC APPARATUS}

The gas-puff Z-pinch device has four capacitors in parallel. The schematic drawing of the discharge chamber of the gaspuff Z-pinch device is shown in Fig. 1. The diameter of the

Manuscript received August 7, 1997; revised January 5, 1998. This work was supported by the POSTECH/BSRI Special Fund, 1996, and the Basic Science Research Institute Program, Ministry of Education, 1996, under Project BSRI-96-2439.

The authors are with the Physics Department, Pohang University of Science and Technology, San 31 Hyoja-Dong, Pohang, Kyungbuk 790-784, Korea (e-mail: shkim@anyon.postech.ac.kr).

Publisher Item Identifier S 0093-3813(98)06366-8.

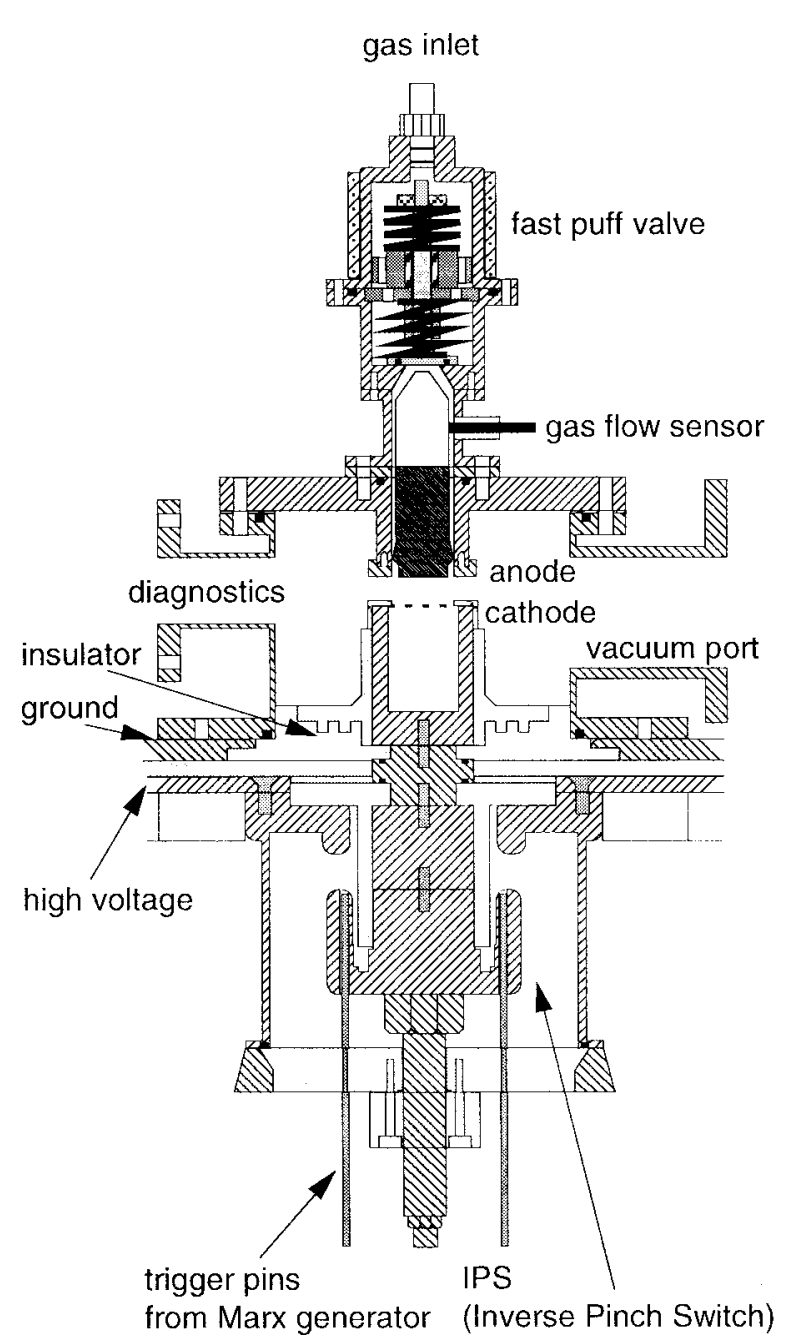

Fig. 1. Schematic drawing of gas-puff Z-pinch device.

inner wall of the chamber is $13.5 \mathrm{~cm}$. The anode is made of copper, the cathode brass, and the other parts stainless steel. The device is pumped down to the pressure of $10^{-6}$ torr by a diffusion pump. A hollow gas shell is puffed through an annular nozzle into the vacuum chamber by opening the fast valve. The diameter of the annular nozzle is $2.8 \mathrm{~cm}$ and the separation of the electrodes is $8 \mathrm{~mm}$. The mass of gas shell can be controlled mainly by changing the time delay between the gas puff and the discharge. The mass load is carefully adjusted so that a pinch occurs when the current reaches its maximum. Argon is used as a working gas. 
For the study of pinched stage with a crystal spectrometer and a pin-hole camera, $8 \mu \mathrm{F}$ capacitors were used, and these were replaced with $11.5 \mu \mathrm{F}$ capacitors for the measurements with an XRD and an XUV spectrometer to increase energy storage. An inverse pinch switch (IPS) was adopted as a main switch of discharge which has many advantages over a conventional spark gap switch [10]. IPS accommodates a large current since its current spreads out due to inverse pinch dynamics and shows small erosion of electrode materials compared with a conventional spark gap switch. Since it is adopted as a single unit, a requirement of simultaneous triggering arising when several spark gaps are used may be avoided. The basic electrical parameters of the gas-puff Z-pinch device were obtained by short-circuit discharge. With $8 \mu \mathrm{F}$ capacitors its inductance is measured to be $51 \mathrm{nH}$ and the rise time of the current is $1.0 \mu \mathrm{sec}$. With $11.5 \mu \mathrm{F}$ capacitors, the inductance of $57 \mathrm{nH}$ is obtained with the rise time of $1.27 \mu \mathrm{sec}$.

In order to monitor the pinch time and the reproducibility of pinches a B-dot probe was placed inside the chamber through a chamber port. The probe is a small acrylic rod of $4 \mathrm{~mm}$ diameter wound with copper wires of $0.3 \mathrm{~mm}$ diameter. The distance of the probe from the central axis of the two electrodes is $59 \mathrm{~mm}$. The calibration of B-dot probe was done through a short-circuit discharge whose result agrees with a calculation using the probe parameters of fabrication. In a cylindrical geometry the induced voltage at this probe is proportional to the change rate of magnetic flux so that we can measure the change of the plasma current $d I / d t$.

An X-ray pin-hole camera was used to image the hightemperature, high-density region of plasma radiating X-rays. A stainless steel pin-hole of $100 \mu \mathrm{m}$ diameter was used and a $5 \mu \mathrm{m}$-thick aluminum foil was placed in front of the pin-hole as a filter which transmits an X-ray whose photon energy is above $0.8 \mathrm{keV}$ (10\% cutoff). Kodak DEF-392 film was used to record the images. The distance from plasma source to the pin-hole is $7.7 \mathrm{~cm}$ and from the pin-hole to the film $6.6 \mathrm{~cm}$.

$\mathrm{X}$-rays emitted from hot spots of pinch stage were recorded using a flat germanium crystal $(2 d=6.5 \AA)$ spectrometer. The spatial resolution along the axis was obtained by placing a horizontal slit of $1 \mathrm{~mm}$ width in the midpoint between source and the crystal. The distance from plasma source to the crystal is $18 \mathrm{~cm}$. The film, Kodak DEF-392, was covered with $4 \mu \mathrm{m}$ thick aluminized Kimfol foil to prevent the exposure by visible lights.

Soft X-ray spectra of gas-puff Z-pinch plasmas were obtained with a $2 \mathrm{~m}$ grazing incidence XUV spectrometer [11]. A 600 lines $/ \mathrm{mm}$ grating was set at a grazing angle of $1.5^{\circ}$ with the entrance slit of $22 \mu \mathrm{m}$ width. A toroidal mirror was placed between the plasma source and the spectrometer to collect and focus the light into the entrance slit and also to correct the astigmatism of the grazing incidence spectrometer. A glass capillary array of $2 \mathrm{~mm}$ thickness was placed in front of the toroidal mirror to protect the optical elements from plasma debris.

The spectrometer was oriented so that the plane of dispersion was perpendicular to the axis of plasma column to spatially resolve the spectra in the axial direction. The distance from plasma source to the center of the toroidal mirror is

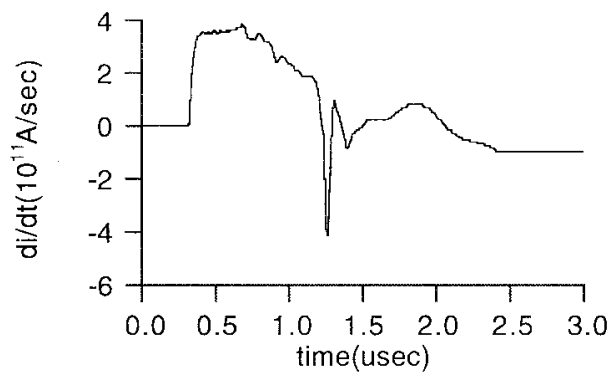

(a)

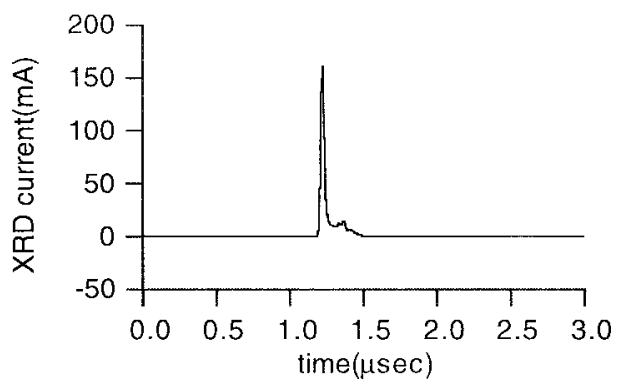

(b)

Fig. 2. $d I / d t$ of gas-puff Z-pinch and current of XRD. (a) $d I / d t$ is measured by B-dot probe for discharge of argon gas-puff Z-pinch with 11.5 $\mu \mathrm{F}$ capacitance and $25 \mathrm{kV}$ charging voltage. The pinch time is $0.95 \mu \mathrm{sec}$ after breakdown. (b) Current signal of XRD is measured with an XRD made of aluminum cathode and filtered by $1.5 \mu \mathrm{m}$ thick mylar. It reaches peak about $50 \mathrm{~ns}$ earlier than that of $d I / d t$ dip signal.

$54.5 \mathrm{~cm}$ and from the mirror to the grating $60.7 \mathrm{~cm}$. Timeintegrated soft X-ray emissions were recorded on the Kodak 101-05 photographic plates.

An XRD was used to acquire the time-evolution of soft Xray emissions. It consists of a filter, a nickel mesh anode of $75 \%$ transmittance, and an aluminum photocathode which is biased to $-400 \mathrm{~V}$. The cathode material was prepared similarly to R. H. Day et al. [12]. Thus we used the quantum efficiency data measured by them. The entrance hole of XRD is 17.9 $\mathrm{cm}$ distant apart from plasma source and $6 \mathrm{~mm}$ in diameter, and a stainless steel mesh of $28 \%$ transmittance was inserted to reduce the intensity of the incident light. A $1.5 \mu \mathrm{m}$-thick mylar $\left(\mathrm{C}_{10} \mathrm{H}_{8} \mathrm{O}_{4}\right)$ sheet was used as a filter with which the photocathode sensitively responds to soft X-rays in the spectral region of 44 to $110 \AA$. XRD yields a photoelectric current responding to incident radiation. The integration of this current provides us with the total charge of electrons ejected.

\section{Hot Spots of Pinch Plasma}

In this experiment we charged the capacitors of $8 \mu \mathrm{F}$ up to the voltages of $-25 \mathrm{kV}$ to $-30 \mathrm{kV}$. The current rose within $1.0 \mu \mathrm{sec}$ to about $300 \mathrm{kA}$ at pinch. After puffing argon gas, we adjusted the delay time between gas puffing and the main discharge in order to obtain a pinch at current maximum. The pinch appears as an abrupt dip of about $100 \mathrm{~ns}$ width in the B-dot probe signal as shown in Fig. 2(a). Shot-toshot variation of the pinch time is within $10 \%$. Hot spots are formed in the pinch stage. This is shown by the X-ray pin-hole photography (Fig. 3). In Fig. 3(b), four to five spots with the size of about $400 \mu \mathrm{m}$ diameter appeared accompanying plasma 


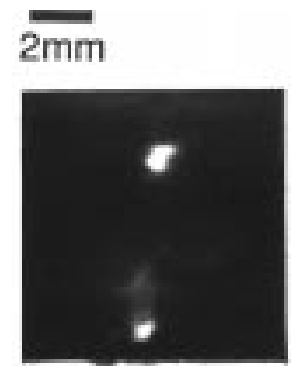

(a)

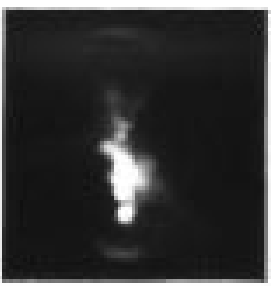

(b)

Fig. 3. X-ray pin-hole pictures $(\times 3$ enlarged $)$ of hot spots. Top side of the picture corresponds to anode and it is taken through $5 \mu \mathrm{m}$-thick aluminum filter. (a) Two spots emitting X-rays and (b) shows more complicated shapes. Discharges were done with $8 \mu \mathrm{F}$ capacitance and charging voltages $-25 \mathrm{kV}$ in (a) and $-30 \mathrm{kV}$ in (b)

clouds. These spots have been considered to be initiated by sausage instabilities in a cylindrical Z-pinch type plasma. In our experiment the number of spots and their positions were not regular, but they were localized within a radius less than $1 \mathrm{~mm}$ from the central axis of the electrodes and their sizes were a few-hundred $\mu \mathrm{m}$ in diameter. The pin-hole picture also shows that a plasma is more broadly distributed around the anode than near cathode. He-like resonance lines and Li-like dielectronic satellite lines from the hot spots were recorded with crystal spectrometer.

Fig. 4 shows a resonance line and an intercombination line of He-like Ar XVII and dielectronic satellite $(j k l)$ lines of Lilike Ar XVI for the pinched hot plasmas, whose pin-hole images are shown in Fig. 3. The relative intensities between these lines are similar to others' results [7]. Though several spots were produced as shown in the pin-hole image, the spatiallyresolved spectra indicated that noticeable intensities were contributed mainly from one hot spot. Electron temperature and density can be diagnosed from the intensity ratios of these lines [13], [14]. Even though the dependence of the ratio of the resonance line intensity $\left(I_{R}\right)$ to the intercombination line intensity $\left(I_{I}\right)$ on the temperature and the dependence of the ratio of the satellite line $(j k l)$ intensity $\left(I_{S}\right)$ to $I_{R}$ are weak, the electron density and temperature were determined to satisfy both of the ratios that were experimentally observed. Fig. 5 is the contour plot of the intensity ratios of $I_{R} / I_{I}$ and $I_{S} / I_{R}$ calculated using RATION code [15]. It shows the weak dependence of $I_{R} / I_{I}$ on the temperature and the weak dependence of $I_{S} / I_{R}$ on the density. The electron density and temperature satisfying both ratios are $8 \times 10^{20} \mathrm{~cm}^{-3}$ and $850 \mathrm{eV}$ for Fig. 4(a) and $5 \times 10^{20} \mathrm{~cm}^{-3}$ and $800 \mathrm{eV}$ for Fig. 4(b), as shown in Fig. 5 . A little higher density and temperature were obtained for 25 $\mathrm{kV}$-discharge. In the $25 \mathrm{kV}$-discharge, a pinch occurred at the time of maximum current while in the $30 \mathrm{kV}$-discharge 150
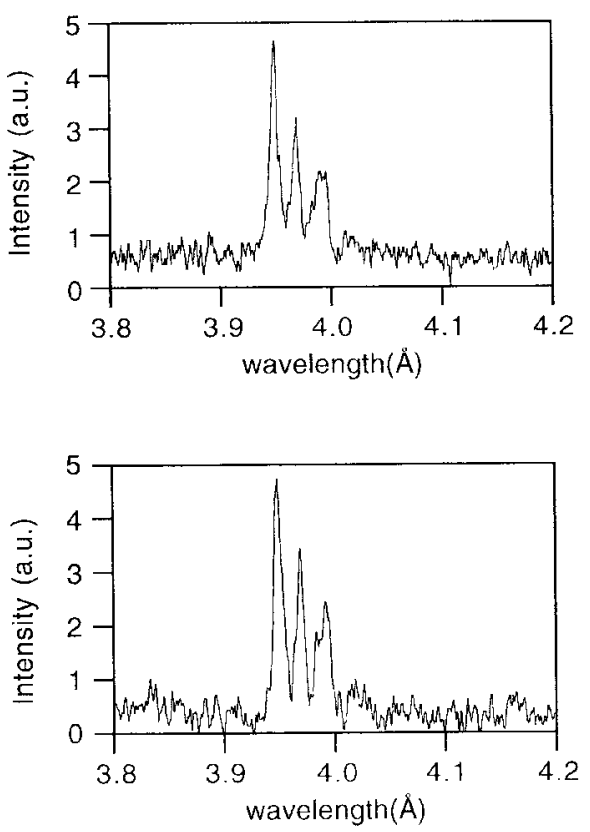

Fig. 4. Lines from Ar XVI and XVII. The left two lines are $1 s 2 p^{1} P-1 s^{21} S_{0}$ (resonance line) and $1 s 2 p^{3} P-1 s^{21} S_{0}$ (intercombination line) of Ar XVII, respectively. The third is dielectronic satellite line $(j k l)$ of Ar XVI. Corresponding pin-hole pictures of each spectrum are shown in Fig. 3.

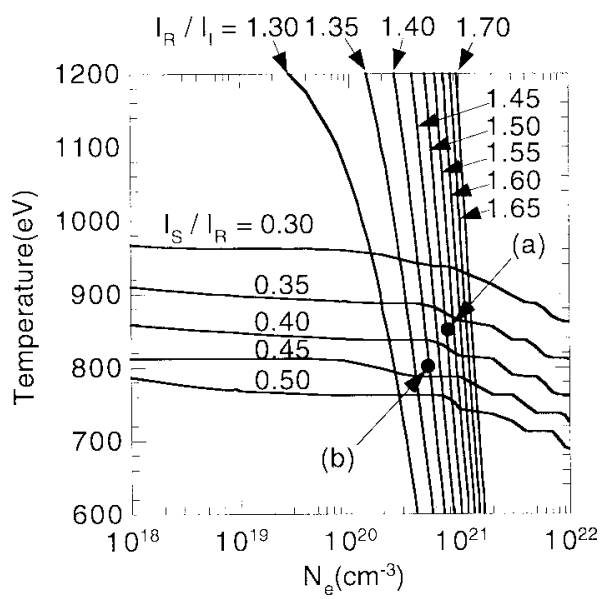

Fig. 5. The contour plot of the intensity ratios of $I_{R} / I_{I}$ and $I_{S} / I_{R}$ of He-like argon. The points marked as (a) and (b) represent the ratios corresponding to the spectra (a) and (b) of Fig. 4, respectively.

ns earlier before pinch. A large discharge voltage or current would not always correspond to a higher temperature. Though the condition where a pinch occurs at the time of maximum current is certainly important, however, a more significant factor may be the pinch-spot state such as the number and sizes of hot spots. In case a lot of spots are generated, spectral intensities radiated from each spot would be smaller.

\section{ESTIMATION OF RADIATION ENERGY}

In this experiment we increased the capacitance to 11.5 $\mu \mathrm{F}$ with a voltage of $-25 \mathrm{kV}$ in order to increase energy storage. A typical $d I / d t$ signal and the soft X-ray output are shown in Fig. 2, where the $d I / d t$ signal is measured by the 


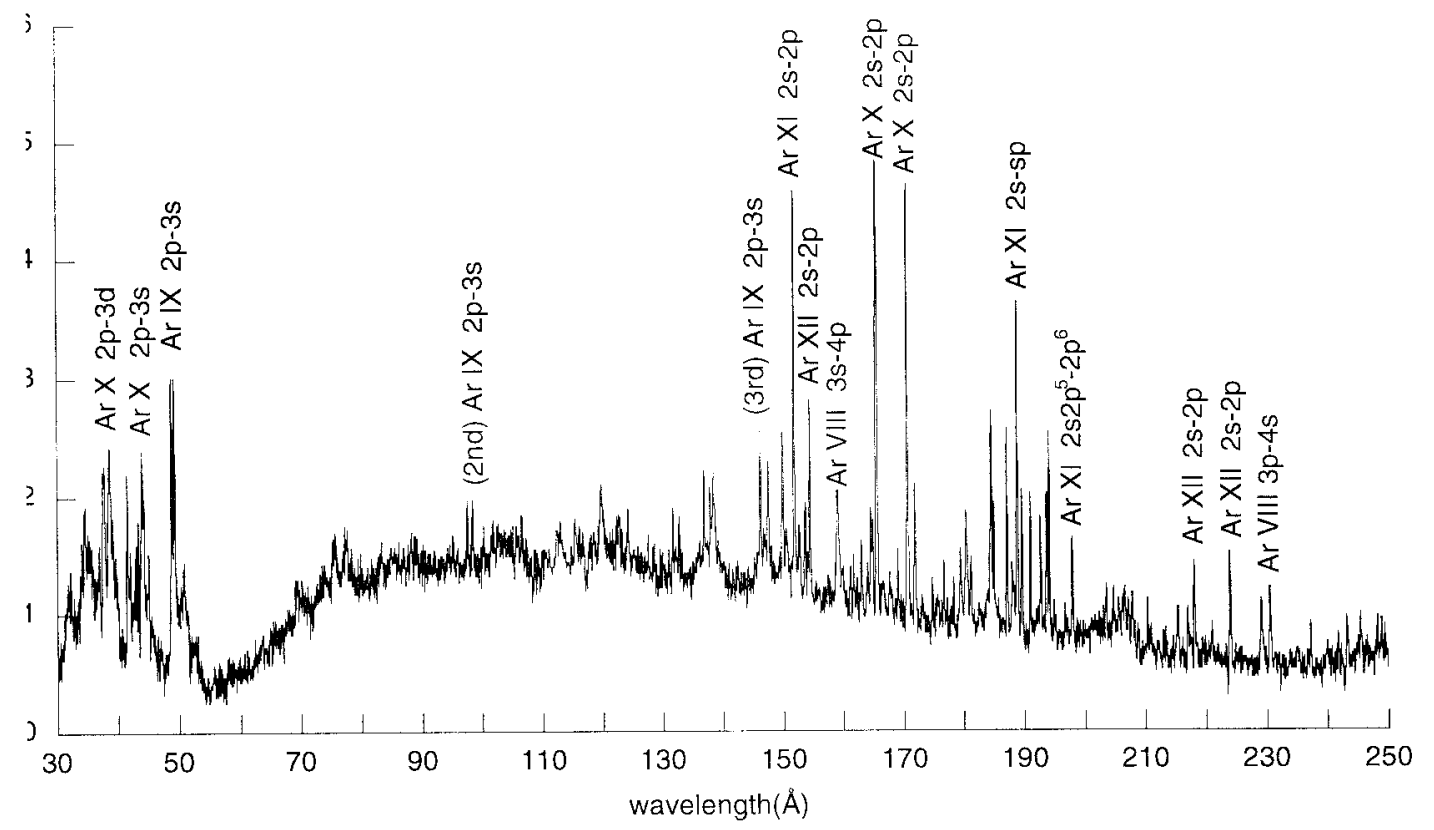

Fig. 6. Spectra of argon gas-puff Z-pinch plasma. The corresponding $d I / d t$ and XRD current are shown in Fig. 2.

B-dot probe and the soft X-ray radiation with XRD. A pinch occurred at $0.95 \mu \mathrm{sec}$ after initial breakdown, which is 0.32 $\mu \mathrm{sec}$ before the quarter period, i.e., $1.27 \mu \mathrm{sec}$. The current at pinch is $260 \mathrm{kA}$. The XRD signal was sensitive to the pinch formation. When the dip in the $d I / d t$ signal was not strong, no XRD output was obtained. The outputs, however, were not much varied when the dip sizes in the $d I / d t$ signals were of the same magnitudes. In Fig. 2, the full-width half-maximum (FWHM) of the soft X-ray pulse is about $40 \mathrm{~ns}$ and the signal peaks about $50 \mathrm{~ns}$ earlier than the time of $d I / d t$ dip, i.e., the maximum compression time. On the contrary, the hard X-ray signal detected by XUV Si-semiconductor photodiode detecting photon energy range above $4.6 \mathrm{keV}$ (10\% cutoff with $36 \mu$ m-thick aluminum filter) reached its peak about 20 ns later than the peak of XRD signal. This implies that soft $\mathrm{X}$-rays are emitted from the beginning of compression and the power reaches its maximum before stagnation, and as the plasma is further compressed and attains higher temperature and density, more intense hard X-rays are emitted.

Using a 2-m grazing incidence XUV spectrometer, a spectrum corresponding to the Fig. 2 was recorded. The developed plate was read by a densitometer. The calibrated optical density was obtained by the use of standard diffuse density wedge and Henke's formula [16]. The intensities of the spectrum varied along the axial direction. The spectrum in the region of 30 to $250 \AA$ is shown in Fig. 6. An intense continuum is present around $120 \AA$, and most of spectral lines are emitted from the ions of Ar VIII to Ar XIII ionization stages. The electron temperature of a plasma radiating lines from these ionization stages is about $150 \mathrm{eV}$ [6]. The electron density estimated from the ratio of the line Ar XI $2 s 2 p^{5}-2 p^{6}$ (197.95 $\AA$ ) to $\mathrm{Ar}$ $\mathrm{XI} 2 s^{2} 2 p^{4}-2 s^{2} p^{5}(171.86 \AA)$ is about $8 \times 10^{18} \mathrm{~cm}^{-3}$ [17].

The radiation output energy can be estimated with the known filter transmission [18], cathode response [12], and the relative spectral intensity profile obtained with the XUV spectrometer. The spectral profile $d W / d E$ in the photon energy range $[E, E+d E]$ incident on a spectrometer results in a modified spectral intensity $d W^{\prime} / d E$

$$
\frac{d W^{\prime}}{d E}=A(E) \frac{d W}{d E}
$$

where $A(E)$ is a sensitivity factor including a grating efficiency. Charge $Q$ collected by an XRD is expressed as

$$
Q=\int\left(\frac{d W}{d E}\right) \cdot S(E) \cdot d E
$$

where $S(E)$ is a response sensitivity of an XRD with a filter. For a relatively narrow region of photon energy, $A(E)$ can be set as a constant. We estimate the radiation energy in the region where the sensitivity of the XRD is high. The absolute radiation energy $W$ can be rewritten as

$$
W=Q \cdot \frac{\int \frac{d W^{\prime}}{d E} \cdot d E}{\int \frac{d W^{\prime}}{d E} \cdot S(E) \cdot d E}
$$

where the integration is done over the energy region where the response of XRD is sensitive. Fig. 7 shows the spectra (Fig. 6) in the energy scale with the response sensitivity of XRD filtered by a $1.5 \mu \mathrm{m}$-thick mylar. The XRD is sensitive in the wavelength range of 44 to $110 \AA$ or in the energy region of 110 to $280 \mathrm{eV}$. Considering transmittance of meshes, and assuming the uniform radiation through $4 \pi$ steradian, we estimated the total radiation energy from Z-pinch source to be 11 Joule in the wavelength range of 44 to $110 \AA$ into $4 \pi$ steradian for the measurement of XRD in Fig. 2(b). Assuming uniform efficiency in the wavelength range of 30 to $250 \AA$ for a rough estimation, the above result implies that the output energy in the range would be 23 Joule which is about $0.6 \%$ of the stored electric energy ( $3.6 \mathrm{kJoule}$ ). In another experiment done after some modifications on the device (nozzle shape and switch), the radiated energy of up to 206 Joule was estimated 


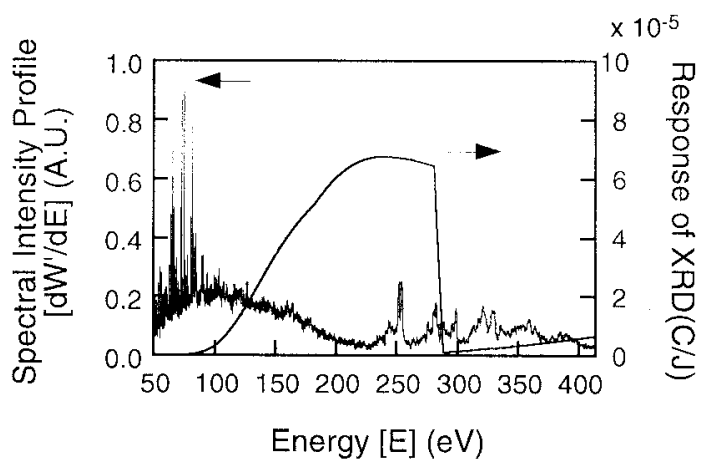

Fig. 7. Spectral intensity profile and response sensitivity of XRD. The spectra in Fig. 6 are shown in the energy scale with the response sensitivity of aluminum XRD filtered by $1.5 \mu \mathrm{m}$-thick mylar.

in the spectral range of 30 to $250 \AA$, for which a separate paper is under preparation.

\section{CONCLUSION}

The radiation characteristics of a gas-puff Z-pinch argon plasma has been studied. Diagnostics of hot spots at pinch using a crystal spectrometer shows that its electron temperature and density are about $1 \mathrm{keV}$ and $10^{21} \mathrm{~cm}^{-3}$, respectively. The XUV spectra show that the device can be a good source for the spectroscopy and X-ray applications since it emits spectra of highly-ionized ions and its radiation output is efficient to produce copious soft X-rays. We estimated the radiation output of 11 Joule in the wavelength range of 44 to $110 \AA$ into $4 \pi$ steradian.

\section{REFERENCES}

[1] J. S. Perlman, J. C. Riordan, and A. C. Kolb, "A bright pulsed Xray source for soft X-ray research and processing applications," Radiat. Phys. Chem., vol. 25, no. 4-6, pp. 709-718, 1985.

[2] H.-J. Shin, D.-E. Kim, and T.-N. Lee, "Soft X-ray amplification in a capillary discharge," Phys. Rev. E, vol. 50, no. 2, pp. 1376-1382, Aug. 1994.

[3] T. Wagner, E. Eberl, K. Frank, W. Hartmann, D. H. H. Hoffmann, and R. Tkotz, "XUV amplification in a recombining Z-pinch plasma," Phys. Rev. Lett., vol. 76, no. 17, pp. 3124-3127, Apr. 1996.

[4] N. R. Pereira and J. Davis, "X-rays from Z-pinches on relativistic electron-beam generator," J. Appl. Phys., vol. 64, no. 3, pp. R1-R27, Aug. 1988.

[5] P. G. Burkhalter, J. Shiloh, A. Fisher, and R. D. Cowan, "X-ray spectra from a gas-puff Z-pinch device," J. Appl. Phys., vol. 50, no. 7, pp. 4532-4540, July 1979.

[6] R. E. Marrs, D. D. Dietrich, R. J. Fortner, M. A. Levine, D. F. Price, R. E. Stewart, and B. K. F. Young, "Time and space resolved vacuumultraviolet spectroscopy of an argon gas-puff Z-pinch," Appl. Phys. Lett., vol. 42, no. 11, pp. 946-948, June 1983.

[7] G. Nave, C. D. Challis, A. E. Dangor, and J. G. Lunney, "Soft X-ray spectra from a gas-puff Z-pinch," J. Appl. Phys., vol. 65, no. 9, pp. 3385-3390, May 1989.

[8] J. D. Hares, R. E. Marrs, and R. J. Fortner, "An absolute measure of heating by suprathermal electrons in a gas puff Z-pinch," J. Phys. D, vol. 18, no. 4, pp. 627-631, Apr. 1985.

[9] J. Bailey, "Effect of radiation cooling and plasma atomic number on Z-pinch dynamics," Ph.D. dissertation, Univ. California, Irvine, 1984.

[10] K. S. Han and J. H. Lee, "Comparative study of inpistron and spark gap," in Proc. 1992 20th Power Modulator Symp., Myrtle Beach, SC, June 1992, pp. 402-405.

[11] J. L. Schwob, A. W. Wouters, S. Suckewer, and M. Finkenthal, "High-resolution duo-multichannel soft X-ray spectrometer for Tokamak plasma diagnostics," Rev. Sci. Instrum., vol. 58, no. 9, pp. 1601-1615, Sept. 1987.

[12] R. H. Day, P. Lee, E. B. Saloman, and D. J. Nagel, "Photoelectric quantum efficiencies and filter window absorption coefficients from 20 eV to $10 \mathrm{keV,"} \mathrm{J.} \mathrm{Appl.} \mathrm{Phys.,} \mathrm{vol.} \mathrm{52,} \mathrm{no.} \mathrm{11,} \mathrm{pp.} \mathrm{6965-6973,} \mathrm{Nov.}$ 1981.

[13] A. H. Gabriel, "Dielectronic satellite spectra for highly-charged He-like ion lines," Mon. Not. R. Astr. Soc., vol. 160, pp. 99-119, 1972.

[14] I. Yu. Skobelev, A. V. Vinogradov, and E. A. Yukov, "Densitydependent lines of one- and two-electron ions in diagnostics of laboratory plasma. II. Intensity line ratios of hydrogen-like, helium-like, and oxygen-like multicharged ions," Phys. Scr., vol. 18, no. 2, pp. 78-86, Aug. 1978

[15] R. W. Lee, B. L. Whitten, and R. E. Strout, "Specra-A model for Kshell spectroscopy," J. Quant. Spectrosc. Radiat. Transfer., vol. 32, pp. 91-101, 1984.

[16] B. L. Henke, F. G. Fujiwara, M. A. Tester, C. H. Dittmore, and M. A. Palmer, "Low-energy x-ray response of photographic films. II. Experimenta," J. Opt. Soc. Amer. B, Opt. Phys., vol. 1, no. 6, pp. 828-849, Dec. 1984

[17] U. Feldman and G. A. Doschek, "Plasma diagnostics using highresolution spectroscopic techniques," J. Opt. Soc. Amer., vol. 67, no. 6, pp. 726-734, June 1977.

[18] B. L. Henke, E. M. Gullikson, and J. C. Davis, "X-ray interactions: Photoabsorption, scattering, transmission, and reflection at $E=50$ 30000 eV, $Z=1-92$," Atomic Data Nucl. Data Tables, vol. 54, no. 2 , pp. 181-342, July 1993.

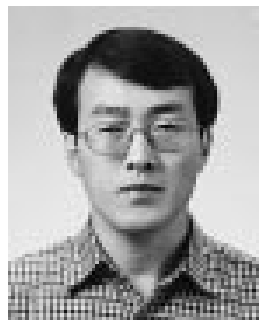

Seong Ho Kim was born in Kyungbuk, Korea, on July 15,1963 . He received the degree in physics from Seoul National University, Korea, in 1990 and is currently pursuing the Ph.D. degree in the Physics Department at Pohang University of Science and Technology, Korea.

His research is concentrated on the spectroscopic study of gas-puff Z-pinch plasma.

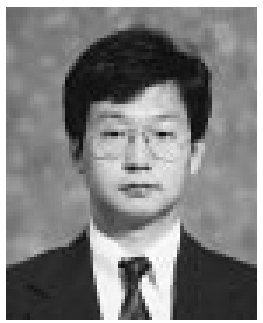

Dong-Eon Kim received the Ph.D. degree from Princeton University, Princeton, NJ, in 1989.

In 1991, he joined the faculty in the Physics Department at Pohang University of Science and Technology, Korea. His research interest has been the development and the study of dynamics of X-ray radiation sources and their applications: soft X-ray laser experiments using a fast high-power capillary discharge, gas-puff Z-pinch plasma experiments, and multilayer soft X-ray reflector development using a pulsed-laser deposition.

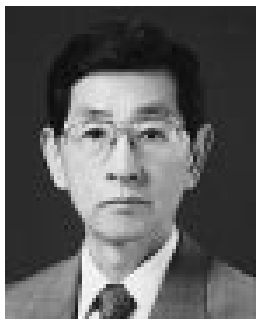

Tong Nyong Lee received the Ph.D. degree in nuclear physics from the University of London, U.K., in 1959.

He served as a faculty member at the Seoul National University, Korea, from 1960 to 1964, and the Catholic University of America, Washington, DC, from 1964 to 1970 . He was then employed at the United States Naval Research Laboratory as a Research Physicist before accepting a faculty position at Pohang University of Science and Technology (POSTECH), Korea, in 1988. His main interest areas are high density, high temperature plasmas, and X-ray laser research. He is now a Professor Emeritus at POSTECH. 